ELSO The ELSO database contains 78 patients (adult, 59; paediatric, 19) with pulmonary vasculitides who received ECMO. 43 had a diagnosis of Granulomatosis and Polyangiitis (GPA), whereas the remaining diagnoses included hypersensitivity angiitis, Goodpasture's syndrome and thrombotic microangiopathy. The median age was 23 yrs (IQR 16-47). The median duration of ECMO was $190 \mathrm{hrs}$ (IQR 146-282) and ICU survival was $82 \%$. Twelve patients $(15 \%)$ were reported to have thrombotic ECMO circuit complications.

Conclusion In this case series, ECMO offers an excellent survival rate in SRF due to ANCA-associated DAH. ELSO registry data supports this, suggesting that ECMO should be considered as supportive therapy in DAH with SRF not responsive to conventional therapy.

\section{P279 REDUCTION IN DISEASE PROGRESSION WITH NINTEDANIB IN THE INPULSISTM TRIALS}

${ }^{1} V$ Cottin, ${ }^{2} \mathrm{H}$ Taniguchi, ${ }^{3} \mathrm{HR}$ Collard, ${ }^{4} \mathrm{~L}$ Richeldi, ${ }^{5} \mathrm{~S}$ Stowasser, ${ }^{6} \mathrm{~T}$ Tschoepe, ${ }^{7} \mathrm{R}$ SchlenkerHerceg, ${ }^{8} \mathrm{G}$ Raghu. 'Louis Pradel Hospital, University of Lyon, Lyon, France; ${ }^{2}$ Tosei General Hospital, Aichi, Japan; ${ }^{3}$ University of California San Francisco, San Francisco, California, USA; ${ }^{4}$ University of Southampton, Southampton, UK; ${ }^{5}$ Boehringer Ingelheim Pharma GmbH and Co. KG, Ingelheim Am Rhein, Germany; ${ }^{6}$ Boehringer Ingelheim France S. A. S., Reims, France; 'Boehringer Ingelheim Pharmaceuticals Inc., Ridgefield, Connecticut, USA; ${ }^{8}$ University of Washington, Seattle, Washington, USA

\subsection{6/thoraxjnl-2014-206260.397}

Background Nintedanib, an intracellular inhibitor of tyrosine kinases, is in development for the treatment of idiopathic pulmonary fibrosis (IPF). The INPULSIS ${ }^{\mathrm{TM}}$ trials were two replicate 52week, randomised, double-blind, placebo-controlled Phase III trials that investigated the efficacy and safety of nintedanib $150 \mathrm{mg}$ twice daily in 1066 patients with IPF. Declines in forced vital capacity (FVC) $\%$ predicted of $>5 \%$ and $>10 \%$ in patients with IPF have been proposed as indicators of disease progression and have been associated with reduced survival.

Aim To determine the effect of nintedanib on changes in $\mathrm{FVC} \%$ predicted in the INPULSIS ${ }^{\mathrm{TM}}$ trials.

Methods The proportions of patients with absolute and relative declines in $\mathrm{FVC} \%$ predicted of $>5 \%$ and $>10 \%$ at week 52 in each INPULSIS $^{\mathrm{TM}}$ trial were determined in a post-hoc analysis.

Results In each trial, a significantly greater proportion of patients in the placebo group had an absolute decline in FVC\% predicted of $>5 \%$ compared with the nintedanib group. In INPULSIS $^{\mathrm{TM}}-1$, a significantly greater proportion of patients in the placebo group had an absolute decline in $\mathrm{FVC} \%$ predicted of $>10 \%$ compared with the nintedanib group; the difference between groups in INPULSIS ${ }^{\mathrm{TM}}-2$ was numerically in favour of nintedanib but did not reach statistical significance. In each trial, significantly greater proportions of patients in the placebo group had relative declines in $\mathrm{FVC} \%$ predicted of $>5 \%$ and $>10 \%$ compared with the nintedanib group.

Conclusion In the INPULSIS ${ }^{\text {тм }}$ trials, nintedanib reduced the proportion of patients with IPF who experienced disease progression as measured by categorical FVC decline.

\section{P280 EXTENDED CLINICAL EXPERIENCE WITH PIRFENIDONE DURING A NAMED PATIENT PROGRAMME FOR IDIOPATHIC PULMONARY FIBROSIS (IPF): INTERIM RESULTS}

${ }^{1} \mathrm{H}$ Parfrey, ${ }^{2} \mathrm{~N}$ Chaudhuri, ${ }^{3} \mathrm{MA}$ Gibbons, ${ }^{3} \mathrm{~L}$ Anning, ${ }^{4} \mathrm{M}$ Balkin, ${ }^{5} \mathrm{~S}$ Cooper, ${ }^{5} \mathrm{R}$ Dew, ${ }^{4} \mathrm{TM}$ Maher. 'Papworth Hospital NHS Foundation Trust, Cambridge, UK; ${ }^{3}$ Royal Devon and
Exeter NHS Foundation Trust, Exeter, UK; ${ }^{4}$ Royal Brompton and Harefield NHS Foundation Trust, London, UK; ${ }^{5} \mathrm{pH}$ Associates Ltd, Marlow, UK; ${ }^{2}$ University Hospital of South Manchester NHS Foundation Trust, Manchester, UK

\subsection{6/thoraxjnl-2014-206260.398}

Introduction and objectives From September 2011 to May 2013, pirfenidone was available in the UK in a named patient programme (NPP). We present results from an extension to a previous real-world study (Parfrey et al. Abstract S98, BTS Winter Conference 2012) now including longer follow-up and all patients enrolled in the pirfenidone NPP from 4 centres.

Methods Four centre, retrospective, cohort review of patient outcomes in the 24 months following pirfenidone initiation in the NPP. Discontinuation data were separately collected for all patients prescribed pirfenidone at the Brompton between Sept 2011 and May 2014.

Results Two hundred and eighteen eligible patients have been identified. Demographic data have been collected for 124 patients (78\% male) and outcome data at 12 months from 58 patients. Mean $( \pm$ S. D.) age at diagnosis was $67.1( \pm 8.1)$ years. Mean time from diagnosis to pirfenidone initiation was 27.7 ( \pm 30.6) months. At pirfenidone initiation, mean FVC was 69.3 $( \pm 18.9) \%$ predicted (with $27(22.0 \%)$ patients having FVC $>80 \%$ predicted); DLco was $40.3( \pm 13.8) \%$ predicted. Following a 14-day titration period, 53 (93\%) patients were receiving the recommended dose of $2403 \mathrm{mg} /$ day pirfenidone. At 6 and 12 months; $47(81 \%)$ and $44(76 \%)$ patients continued to receive pirfenidone.

187 patients have been prescribed pirfenidone at the Brompton since Sept 2011. At 10 months following initiation 18.5\% of these have discontinued pirfenidone with no further discontinuations beyond this time. For patients in the NPP with available paired baseline and 6 or 12 month FVC, mean decline in FVC\% predicted over first 6 months of pirfenidone treatment was 3.2 $( \pm 7.9) \%$; over first 12 months $1.6( \pm 12.0) \%$. One patient's FVC $\%$ predicted declined $>10 \%$ in the first 12 months of treatment. Mean decline in DLco\% predicted over first 6 months from pirfenidone initiation was 9.1( \pm 14.7$) \%$; over first 12 months' treatment, $7.0( \pm 17.2) \%$.

Conclusions The high proportion of patients remaining on pirfenidone at 12 months suggests it is well tolerated and any tolerability issues tend to occur early in treatment. Lung function is largely preserved at 12 months following pirfenidone initiation. Longer-term observation of lung function and clinical outcomes will continue to determine the real-world benefits of pirfenidone.

\section{Smoking detection and cessation and non tobacco products}

\section{\begin{tabular}{|l|l}
\hline P281 SMOKING PREVALENCE AND STOP SMOKING \\
\hline
\end{tabular} INTERVENTIONS FOR PATIENTS ADMITTED TO AN EMERGENCY DEPARTMENT (ED) IN A BUSY, INNER CITY HOSPITAL}

${ }^{1} \mathrm{R}$ Thomas, ${ }^{1} \mathrm{~F}$ Warden, ${ }^{2} \mathrm{M}$ Stern. ${ }^{1}$ University of London Medical School, London, UK; ${ }^{2}$ Department Respiratory Medicine, Whittington Health, London, UK

\subsection{6/thoraxinl-2014-206260.399}

Introduction ED admissions are 'teachable moments' to offer cessation advice to smokers. In this study, smoking prevalence and stop smoking interventions were investigated in patients 